\title{
A Multiobjective Particle Swarm Optimization Algorithm for Load Scheduling in Electric Smelting Furnaces
}

\author{
Weijian Kong, Jinliang Ding, Tianyou Chai and \\ Xiuping Zheng \\ State Key Lab of Synthetical Automation for Process \\ Industries \\ Northeastern University \\ Shenyang, China \\ jlding@mail.neu.edu.cn,weijian.kong9@gmail.com
}

\author{
Shengxiang Yang \\ School of Computer Science and Informatics \\ De Montfort University \\ Leicester, UK \\ syang@dmu.ac.uk
}

\begin{abstract}
Electric smelting furnaces, applied in the smelting process of infusible mineral, are highly energy-intensive. In China, they waste a huge amount of electric energy, but yield a small quantity of valuable metals due to the lack of optimized load scheduling strategies. In this paper, we design a multiobjective load scheduling method to minimize the electricity cost and maximize the production output and product quality. Firstly a load scheduling model is developed based on a least square support vector machine, which is a robust empirical model with simple formulation and low sensitivity to external disturbance. We utilize a modified multiobjective particle swarm optimization algorithm to solve the optimization model. The proposed algorithm adopts a supervised population initialization that reuses the past optimal solutions and digs out new candidate solutions to guide the current optimization. An elaborate constraint-handing strategy is devised, which repairs the infeasible solutions that violate the maximum demand constraint and reserve the ones that violate one production constraint but performing excellently on the other production targets. The case study on a typical magnesia-smelting plant shows that the proposed multi-objective load scheduling model and algorithm can achieve an increase of about $14.5 \%$ in the production output, an improvement of about $0.46 \%$ of the magnesia in the product, and a slight saving in electricity cost.
\end{abstract}

Keywords-electric smelting furnaces; load scheduling; multiobjective particle swarm optimization; least square support vector machine

\section{INTRODUCTION}

In many developing countries, the industrial sector accounts for a significant proportion of the total electricity consumption, e.g., over $70 \%$ in China. It is also responsible for the severe energy shortage and peak demand deficit during the peak period of electricity consumption. In order to encourage the load management (LM) on the demand side, utilities have

This work was supported in part by the National Basic Research Program of China under Grant 2009CB320601, NSFC Project under Grant 61273031, 61134006, Liaoning Excellent Talents Project under Grant LJQ2012020, the NKTRD Project 2012BAF19G00 the UK EPSRC under Grant EP/K001310/1, and the Program for New Century Excellent Talents in University. structured electricity tariffs to include time-of-use (TOU) and/or maximum demand (MD) charges. Industries can reduce their electricity costs by adopting appropriate LM strategies in the production process. Several LM strategies based on artificial intelligence (AI) techniques have been proposed for industrial processes. For example, fuzzy logic [1] and neural network [2] are used to shift electricity demand from peak periods to off-peak demand periods in a photovoltaic panel and a petrochemical plant, respectively. However, traditional optimization technique seems to be a better option in addressing the LM problem. A generic load scheduling model for process industries with an optimization formulation was proposed to help them decide the optimal LM strategies [3-4]. Ashok [5] proposed a peak-load management strategy in steel plants, which utilizes integer programming for minimizing the total electricity cost. Babu and Ashok [6] presented an optimization model for LM in the electrolytic process, which adopts a mixed integer nonlinear programming (MINLP) technique to solve the optimization problem. Other studies can be found in [7-8], where the LM problem is addressed in industrial cogeneration and water pumping, respectively.

It should be noted that LM strategies adopted also affect the production target and the operation of industrial processes. For example, a power-curtailment action may result in a diminished production output and temporarily unstable process. As a consequence, some decision-makers hope to simultaneously optimize multiple conflicting objectives, including maximizing the production output and minimizing the electricity cost in the design of LM strategies. The research of multiobjective LM in industrial processes is still rare. However, there have been some investigations in other factors, e.g. distribution of utilities, in the electricity market. In [9-10], a multiobjective decision support model in LM of distribution utilities was proposed, which allows reducing the peak demand, maximizing the utility profit, and minimizing the discomfort caused to consumers. In [10], an interactive method, called STEM [11], has been adopted to provide decision support in selecting remote load control strategies. Gomes et al. [12] adopted an evolutionary algorithm (EA) to 
solve the multiobjective optimization problem and showed the advantages of population-based nature-inspired search algorithms in LM programs. In [13], an interactive EA was adopted for the identification and selection of direct load control actions in electrical distribution networks.

An electric smelting furnace, also called submerged arc furnace or electric arc resistance furnace, is mainly applied in the smelting of non-ferrous metals, e.g., ferroalloys, calcium carbide, and yellow phosphorus [14]. In the furnace, electric current is conducted to the slag or charge through electrodes, and the voltaic arc is generated at the interface between electrode and slag or charge. Since an electric smelting furnace converts electric energy to heat for smelting the infusible mineral, it is highly energy intensive. Usually, a group of furnaces operate in parallel in a plant. So, the total power demand will be huge when most of the furnaces consume a peak power at the same period. To avoid exceeding the contracted MD (CMD) and save the energy cost, it is necessary to select some furnaces to cut down their power consumption. As the power consumption has a crucial role in the production output of a furnace, the design of LM strategy should allow both meeting the production target and reducing the electricity cost.

In this paper, we present a multiobjective load scheduling model for smelting furnaces, in which the decision variables consist of the power consumption by each furnace during each time interval, and the objectives to be optimized are the total production output, average quality, and electricity cost. A least square support vector machine (LS-SVM) is used to estimate the functions of production output and quality, respectively. For solving the multiobjective load scheduling problem, a Pareto- dominance based particle swarm optimization (PSO) approach is proposed to search for a non-dominated solution set, each element of which represents a load scheduling program. Decision-makers can then select a solution from the set that most satisfies their preference, and obtain a load scheduling result that determines the optimal power consumption by each furnace in each period. While smelting mineral, an automatic control mechanism (ACM) is used to regulate electrodes to set the actual power consumed by each furnace to the level according to the load scheduling result.

The rest of this paper is organized as follows. Section II presents the mathematical formulation of the multiobjective load scheduling problem for electric smelting furnaces. Section III is devoted to describe the proposed multiobjective PSO algo- rithm for solving the modeled multiobjective load scheduling problem. The experimental study is presented in Section IV. Finally, Section V concludes this paper.

\section{THE MULTIOBJECTIVE MODEL}

A group of electric smelting furnaces are utilized to simultaneously smelt mineral in a plant. In the design of the load scheduling mechanism, two factors will be considered here.

First, the energy efficiency and facility capacity are different for different furnaces. Second, the power demand of a furnace will change because the smelting process oscillates on multiple operation modes, such as charging, exhausting, and normal operation. The trigger moment and duration time of each operation mode are pre-specified. The load scheduling mecha- nism is illustrated in Fig. 1. The load scheduling provides a series of set points (i.e. the optimal power consumption values by each furnace in each time interval) and then ACM maintains the actual consumption at the level of provided set points.

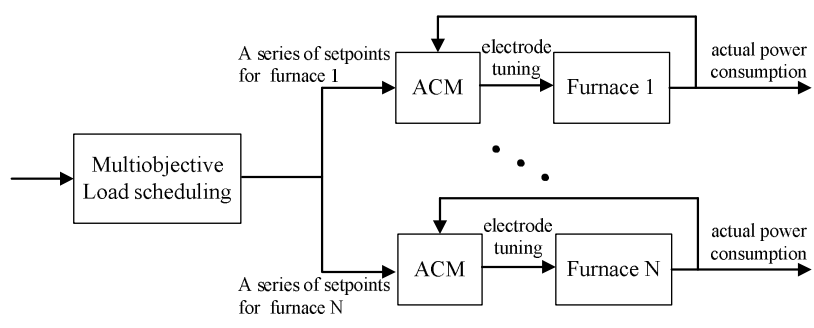

Fig. 1. The load scheduling mechanism.

As the electric smelting process is accompanied by various complex physical and chemical reactions, it is difficult to build a mechanistic model for the functions of production output or product quality. Nevertheless, plenty of historic process data are available and can be used to train a robust empirical model based on AI techniques. Although the empirical model is not absolutely accurate, it has a low sensitivity to external disturbance and is suitable to perform optimization or control work due to its simplicity. As a novel AI technique, the LS-SVM [15] is a strong machine learning technique and has been widely adopted in regression and classification problems. Some researchers have reported the application of LS-SVM in load forecasting [16-17], which is used for time series prediction. In this paper, LS-SVM is used to estimate the nonlinear function relations between the production target and power consumption for furnaces.

\section{A. Mathematical Formulation}

It is necessary to discretize the time horizon (e.g., one day) to simplify the optimization computation of load scheduling. The longer a time interval is, the smaller the dimension of decision variables is. However, the time interval has to be shorter than the demand interval that refers to the period of time in which MD is measured. Therefore, the time horizon $T$ is split into $K$ intervals of equal duration $t_{\mathrm{d}}$ (e.g., 15 minutes). The number $K$ can be computed by

$$
K=T / t_{d}
$$

The decision variables, denoted by $p_{j t}(j=1, \cdots, N, t=1, \cdots K)$, are a series of power consumptions by each furnace in each time interval, where $N$ presents the number of furnaces in a plant. $p_{j t}$ is the average power consumed by the $j$-th furnace in the $t$-th time interval. The ranges of decision variables are determined by the associated facilities and the current operate mode, which are expressed by a group of inequalities as follows

$$
0 \leq p_{j t} \leq P_{j t}, j=1, \cdots N, t=1, \cdots, K
$$


where $P_{j t}$ is a time-varying upper limit.

The objectives to be optimized include the total production output $Y$, the product quality $Q$, and the total electricity cost $E$ in a plant. The total production output is the sum of production output of each furnace in each time interval. The product quality is the percentage of valuable metals in all products. Therefore, the total production output $Y$ and product quality $Q$ in a plant can be figured out as follows

$$
\begin{gathered}
Y=\sum_{j=1}^{N} \sum_{t=1}^{K} y_{j t} \\
Q=\left(\sum_{j=1}^{N} \sum_{t=1}^{K} y_{j t} q_{j t}\right) /\left(\sum_{j=1}^{N} \sum_{t=1}^{K} y_{j t}\right)
\end{gathered}
$$

where $y_{j t}$ is the production output of furnace $j$ in the interval $t$, which has a function of $y_{j t}=f_{j}\left(p_{j t}, s_{j t}\right)$ based on an LS-SVM estimation, $s_{j t}$ is the operation mode in time interval $t$, and $s_{j t}=1,2,3$ denotes the charging, exhausting and normal operation mode, respectively. $q_{j t}$ is the percentage of valuable metal in the product of furnace $j$ in time interval $t$, which can also be estimated using an LS-SVM function $q_{j t}=g_{j}\left(p_{j t}, s_{j t}\right)$.

The electricity cost consists of charges for energy consumed and charges for the CMD. However, the CMD is usually subscribed by consumers early in a month, so the CMD charges cannot be reduced even though the MD is lowered by a smart load scheduling strategy. Hence, the total electricity cost $E$ is given by

$$
E=\sum_{j=1}^{N} \sum_{t=1}^{K} \alpha(t) p_{j t}+\alpha_{1} P_{C M D}
$$

where $\alpha(t)$ is the time-of-use price (charge per $\mathrm{kWh}$ ) for the interval $t, P_{C M D}$ is the CMD value, and $\alpha_{1} P_{C M D}$ is a fixed fee for a month.

It should be noted that in the above model it is not encouraged to obtain a lower MD in the above optimization objective since the CMD charge is fixed. In order to make use of the potential of saving the CMD charge, we establish an objective function of future electricity cost with a variable CMD charge. The new function of the electricity cost helps guide the consumers to modify the CMD for the next month, but it has nothing to do with the decision of load scheduling for the current month.

The future electricity cost $E^{\prime}$ is calculated as follows:

$$
E=\sum_{j=1}^{N} \sum_{t=1}^{K} \alpha(t) p_{j t}+\alpha_{1} P_{M D}
$$

$$
P_{M D}=\underset{t=1, \cdots, K}{\operatorname{aax}}:\left\{\sum_{j=1}^{N} p_{j t}\right\}
$$

In the smelting furnaces, there are no flow and storage constraints referred to in [4-5]. The constraints here mainly include the production constraints and the MD constraint, which are respectively expressed by

$$
\begin{gathered}
Y \geq Y_{l} \\
Q \geq Q_{l} \\
P_{M D} \leq P_{C M D}
\end{gathered}
$$

where $Y_{l}$ and $Q_{l}$ are the specified minimum production targets, and $P_{C M D}$ is as defined before.

To summarize, the proposed multiobjective load scheduling model is thus given as follows:

$$
\begin{aligned}
\text { Min }: & E, \operatorname{Max}: Y, \operatorname{Max}: Q \\
\text { s.t.: } & =\alpha(t) \sum_{j=1}^{N} \sum_{t=1}^{K} p_{j t}+\alpha_{1} P_{C M D} \\
Y & =\sum_{j=1}^{N} \sum_{t=1}^{K} y_{j t} \\
Q & =\left(\sum_{j=1}^{N} \sum_{t=1}^{K} y_{j t} q_{j t}\right) / \sum_{j=1}^{N} \sum_{t=1}^{K} y_{j t} \\
Y & \geq Y_{l}, Q \geq Q_{l} \\
P_{\text {CMD }} & \geq \operatorname{Max}_{t=1, \cdots, K}:\left\{\sum_{j=1}^{N} p_{j t}\right\} \\
0 & \leq p_{j t} \leq P_{j t}, j=1, \cdots N, t=1, \cdots K
\end{aligned}
$$

In order to find a more economical CMD, the multiobjective load scheduling model with variable CMD is shown as follows:

$$
\begin{aligned}
\text { Min }: & E^{\prime}, \text { Max }: Y, \text { Max }: Q \\
\text { s.t. }: & E^{\prime}=\alpha(t) \sum_{j=1}^{N} \sum_{t=1}^{K} p_{j t}+\alpha_{1} P_{M D} \\
Y & =\sum_{j=1}^{N} \sum_{t=1}^{K} y_{j t} \\
Q & =\left(\sum_{j=1}^{N} \sum_{t=1}^{K} y_{j t} q_{j t}\right) / \sum_{j=1}^{N} \sum_{t=1}^{K} y_{j t} \\
P_{M D} & =\operatorname{Max}_{t=1, \cdots, K}:\left\{\sum_{j=1}^{N} p_{j t}\right\} \\
Y & \geq Y_{l}, Q \geq Q_{l}, P_{M D} \leq P_{C M D} \\
0 & \leq p_{j t} \leq P_{j t}, j=1, \cdots N, t=1, \cdots K
\end{aligned}
$$

where $P_{M D}$ is the MD value that is calculated by 


\section{B. Function Estimation Based on LS-SVM}

An LS-SVM technique is used to estimate the functions of production output and product quality of $N$ furnaces, respectively. Let's take the production output function of the $j$ th furnace as an example. First, we update the training data set by sampling data from the latest smelting process. The input data is $x_{j l}=\left[p_{j l}, s_{j l}\right]$, where $p_{j l}$ is the power demand and $s_{j l}$ is the operation mode at the $l$ th sampling point. The output data is the corresponding production output $y_{j l}$. A training data set $D=\left\{\left(x_{j 1}, y_{j 1}\right), \ldots,\left(x_{j n}, y_{j n}\right)\right\}$ is then obtained with $n$ sampling points.

The training of the LS-SVM model by the data set can be regarded as an optimization problem defined as follows:

$$
\begin{aligned}
& \min _{w, \xi} J(w, \xi)=\frac{1}{2}\|w\|^{2}+\frac{1}{2} \gamma \sum_{l=1}^{n} \xi_{j l}^{2} \\
& \text { s.t. } y_{j l}=\left\langle w, \phi\left(x_{j l}\right)\right\rangle+b+\xi_{j l}, l=1, \ldots, n
\end{aligned}
$$

where $\gamma$ and $\xi_{j l}$ represent the relative weight and regression error, respectively, and the function $J(w, \xi)$ represents the structural risk consisting of empirical risk and confidence range. Minimizing $J(w, \xi)$ guarantees the reliability of the model even though the training data is limited.

To solve the above optimization problem, a Lagrange function is constructed as follows:

$$
\begin{aligned}
L(w, \xi, b, \alpha)= & \frac{1}{2}\|\omega\|^{2}+\frac{1}{2} \gamma \sum_{l=1}^{n} \xi_{j l}^{2} \\
& -\sum_{l=1}^{n} \alpha_{j l}\left[\left\langle w, \phi\left(\mathbf{x}_{j l}\right)\right\rangle+b+\xi_{j l}-y_{j l}\right]
\end{aligned}
$$

where $\alpha_{j l}$ are Lagrange multipliers. The Karush-Kuhn-Tucker (KKT) conditions for optimality are given by

$$
\left\{\begin{array}{l}
\frac{\partial L}{\partial w}=\|w\|-\sum_{l=1}^{n} \alpha_{j l} \varphi\left(x_{j l}\right)=0 \\
\frac{\partial L}{\partial \xi}=\gamma \sum_{l=1}^{n} \xi_{j l}-\sum_{l=1}^{n} \alpha_{j l}=\gamma \xi_{j l}-\alpha_{j l}=0 \\
\frac{\partial L}{\partial b}=\sum_{l=1}^{n} \alpha_{j l}=0 \\
\frac{\partial L}{\partial \alpha}=w \varphi\left(x_{j l}\right)+b+\xi_{j l}-y_{j l}=0
\end{array}\right.
$$

After eliminating $w$ and $\xi_{j l}$, we get the following equation:

$$
\left[\begin{array}{cc}
0 & H^{T} \\
1 & \Omega+I / \gamma
\end{array}\right]\left[\begin{array}{l}
b \\
\alpha
\end{array}\right]=\left[\begin{array}{l}
0 \\
y_{j}
\end{array}\right]
$$

where $y_{j}=\left[y_{j 1}, \ldots, y_{j n}\right], H=[1, \ldots, 1], \alpha_{j}=\left[\alpha_{j 1}, \ldots, \alpha_{j n}\right]$ and $\Omega_{l k}=\left\langle\varphi\left(x_{j l}\right)^{T}, \varphi\left(x_{j k}\right)\right\rangle$ for $l, k=1, \ldots, n$. Using the above equation, $\alpha$ and $b$ can be solved.

According to Mercer's condition, there exists a mapping $\varphi$ and an expansion:

$$
K(x, y)=\sum_{i} \varphi_{i}(x) \varphi_{i}(y)
$$

In this paper, we choose a Radial Basis Function (RBF) kernel as follows:

$$
K\left(\mathbf{x}_{j l}, \mathbf{x}_{j k}\right)=\exp \left(-\frac{\left\|\mathbf{x}_{j l}-\mathbf{x}_{j k}\right\|^{2}}{2 \sigma^{2}}\right)
$$

So, the resulting LS-SVM estimation for the production output becomes:

$$
y_{j l}=\sum_{k=1}^{n} \alpha_{j k} K\left(\mathbf{x}_{j l}, \mathbf{x}_{j k}\right)+b, l=1, \ldots, n
$$

where a parameter pair $(\gamma, \sigma)$ needs to be determined by mini- mizing the root mean square of the prediction error for the training data set. An efficient hybrid PSO can be used to search the optimal parameter pair, which can be found in our previous work in [18].

\section{MULTIOBJECTIVE PARTICLE SWARM OPTIMIZATION}

For multiobjective optimization problems (MOPs), we need to find a set of wide-spread and diverse non-dominated solutions instead of a single optimal solution. In comparison with mathematical programming, nature-inspired algorithms, e.g., EAs and PSO algorithms, have some advantages. First, they can find a number of non-dominated solutions in a single run by the distributed search mechanism. Second, they do not require the accurate model information, such as the gradient information. Third, they can quickly provide an approximate optimization result for a very complex problem, which meets the requirements of many industrial processes.

Several multiobjective EAs (MOEAs), such as NSGA2 [19], SPEA [20], and PAES [21], have been successfully applied in many real world problems. Unlike EAs, PSO does not adopt crossover and mutation operators but updates the position of each particle by tracking the current optimal particle. So, it is characterized by few parameters and fast convergence [22-23]. PSO was first introduced to solve MOPs by Moore and Chapman [24]. When extending PSO to solve MOPs, three main issues should be considered [25]: 1) how to preserve the non-dominated solutions found during the search process, 2) how to elect leaders among non-dominated solutions to guide the search of particles, and 3) how to maintain the population diversified. Coello et al. proposed a multiobjective PSO (MOPSO) approach [26], which preserves non-dominated solutions by utilizing a secondary repository 
similar to the adaptive grid of PAES [21], and adopts a special mutation operator to diversify the population.

In this paper, the MOPSO algorithm in [26] is modified with an elaborate population initialization strategy and a constraint handling technique to address the load scheduling problem. The details of MOPSO [26] will not be introduced here, and we focus on the initialization strategy and constraint handing.

\section{A. Population Initialization}

Load scheduling is carried out once every day for electric smelting furnaces. If the smelting facilities and raw materials do not change obviously, the previous optimization result can be used for reference for later optimization process. An optimal solution obtained on the previous day, called old solution here, is actually the most satisfactory solution selected from the nondominated set. So, it is promising to generate more candidate solutions in the regions near to the old solution in the search space, which can serve as initial individuals to guide the next optimization process.

We propose a straightforward individual initialization procedure that considers the trade-off between exploitation and diversification. Suppose we want to generate Pop solutions, the old solution is denoted by $\hat{P}=\left\{\hat{p}_{j t} \mid j=1, \cdots, N t=1, \cdots K\right\}$, and the solutions to be generated are denoted by $P(i)=\left\{p_{j t}^{(i)} \mid j=1, \cdots, N t=1, \cdots K\right\}, i=1, \cdots$, Pop , the initialization procedure is shown in Fig. 2, where the function unifrnd $(A, B)$ generates a uniformly random number between $A$ and $B$.

\section{B. Constraint Handling}

In its original version in [26], MOPSO lacks a constrainthandling mechanism for constrained optimization problems. There are some well-known constraint-handling approaches, such as rejection, penalization [27-28], and repair [29-30]. In this paper, we divide the constraints into two categories: linear hard-constraints and nonlinear soft-constraints. The MD constraint belongs to hard constraint that cannot be violated, and it is linear, so we devise a simple scheme to repair infeasible solutions in terms of the MD constraint. The production targets are nonlinear. So, it is very difficult to repair the solutions that violate the production constraints. However, we still reserve the solutions that violate one production target constraint slightly but perform excellently on other production target.

The constraint handling technique proposed here consists of two phases. The first one takes place after the updating of particles positions and before the evaluation of new positions. It quickly judges whether the new solutions violate MD constraint. If so, the infeasible solutions will be repaired according to the procedure shown in Fig. 3.

After the first phase, all solutions become feasible regarding the $\mathrm{MD}$ constraint. We then evaluate them by calculating their objective values and choose the nondominated solutions based on the Pareto-dominance relation. If some non-dominated solutions violate the production constraints, the second phase of constraint-handling will be implemented.

$$
\begin{aligned}
& \text { For } i=1 \text { to Pop } \\
& \text { for } t=1 \text { to } K \\
& \text { for } j=1 \text { to } N \\
& \text { where } Z=\frac{P_{j t}}{P o p-1} *(i-1) \\
& \text { if } \sum_{j=1}^{N} p_{j t}^{(i)}>P_{C M D} \\
& \text { Set } p_{s t}^{(i)}=P_{C M D}-\sum_{j=1, j \neq s}^{N} p_{j t}^{(i)}
\end{aligned}
$$$$
p_{j t}^{(i)}=\text { unifrnd }\left(\min \left(Z, \hat{p}_{j t}\right), \max \left(Z, \hat{p}_{j t}\right)\right)
$$$$
\text { Generate a random number " } s \text { " between } 1 \text { and } N
$$

Fig. 2. The initialization procedure.

$$
\begin{aligned}
& \text { For } i=1 \text { to Pop } \\
& \text { for } t=1 \text { to } K \\
& \text { if } \sum_{j=1}^{N} p_{j t}^{(i)}>P_{C M D} \\
& s=\text { random () \% random number } \\
& \text { Repair } p_{s t}^{(i)}=P_{C M D}-\sum_{j=1, j \neq s}^{N} p_{j t}^{(i)} \\
& \text { end } \\
& \text { end }
\end{aligned}
$$

Fig. 3. The repair of infeasible solutions.

Assume that the selected non-dominated solution set is $N D$, and $|N D|$ denotes the number of elements in it. An external repository $R E P$ is used to preserve the feasible non-dominated solutions. Sort all non-dominated solutions $p_{j t}^{(i)}(i=1, \cdots,|N D|)$ in the descending order of their evaluations in the production targets, respectively. Let $\operatorname{rank}_{1}\left(p_{j t}^{(i)}\right)$ and $\operatorname{rank}_{2}\left(p_{j t}^{(i)}\right)$ represent the ranking values of solution $p_{j t}^{(i)}$ in the production output and quality objective functions, respectively. We grade the solutions according to their ranking values. The bigger both $B_{1}\left(p_{j t}^{(i)}\right)$ and $B_{2}\left(p_{j t}^{(i)}\right)$ are, the better solution $p_{j t}^{(i)}$ is.

$$
B_{1}\left(p_{j t}^{(i)}\right)= \begin{cases}3 & \operatorname{rank}_{1}\left(p_{j t}^{(i)}\right)<|N D| / 3 \\ 2 & |N D| / 3 \leq \operatorname{rank}_{1}\left(p_{j t}^{(i)}\right)<2|N D| / 3 \\ 1 & 2|N D| / 3 \leq \operatorname{rank}_{1}\left(p_{j t}^{(i)}\right)<|N D|\end{cases}
$$




$$
B_{2}\left(p_{j t}^{(i)}\right)= \begin{cases}3 & \operatorname{rank}_{2}\left(p_{j t}^{(i)}\right)<|N D| / 3 \\ 2 & |N D| / 3 \leq \operatorname{rank}_{2}\left(p_{j t}^{(i)}\right)<2|N D| / 3 \\ 1 & 2|N D| / 3 \leq \operatorname{rank}_{2}\left(p_{j t}^{(i)}\right)<|N D|\end{cases}
$$

Similarly, the constraint-violating extent of solution $p_{j t}^{(i)}$ can be graded as follows:

$$
C_{1}\left(p_{j t}^{(i)}\right)= \begin{cases}0 & Y\left(p_{j t}^{(i)}\right) \geq Y_{l} \\ -1 & 0<Y_{l}-Y\left(p_{j t}^{(i)}\right)<2.5 \% * Y_{l} \\ -2 & 2.5 \% * Y_{l} \leq Y_{l}-Y\left(p_{j t}^{(i)}\right)<5 \% * Y_{l} \\ -3 & 5 \% * Y_{l} \leq Y_{l}-Y\left(p_{j t}^{(i)}\right)<10 \% * Y_{l} \\ -100 & Y_{l}-Y\left(p_{j t}^{(i)}\right) \geq 10 \% * Y_{l}\end{cases}
$$

$$
C_{2}\left(p_{j t}^{(i)}\right)= \begin{cases}0 & Q\left(p_{j t}^{(i)}\right) \geq Q_{l} \\ -1 & 0<Q_{l}-Q\left(p_{j t}^{(i)}\right)<2.5 \% * Q_{l} \\ -2 & 2.5 \% * Q_{l} \leq Q_{l}-Q\left(p_{j t}^{(i)}\right)<5 \% * Q_{l} \\ -3 & 5 \% * Q_{l} \leq Q_{l}-Q\left(p_{j t}^{(i)}\right)<10 \% * Q_{l} \\ -100 & Q_{l}-Q\left(p_{j t}^{(i)}\right) \geq 10 \% * Q_{l}\end{cases}
$$

An evaluation program to judge whether to preserve a solution is shown in Fig. 4.

$$
\begin{aligned}
& \text { For } i=1 \text { to }|N D| \\
& \quad \text { if } B_{1}\left(p_{j t}^{(i)}\right)+B_{2}\left(p_{j t}^{(i)}\right)+C_{1}\left(p_{j t}^{(i)}\right)+C_{2}\left(p_{j t}^{(i)}\right)>0 \\
& \qquad R E P=R E P \cup p_{j t}^{(i)} \\
& \text { end } \\
& \text { End }
\end{aligned}
$$

Fig. 4. The evaluation program to judge whether to preserve a solution.

\section{CASE STUDY}

\section{A. Experimental Setting}

To illustrate the method proposed, we conduct a case study for an electric smelting plant in the province of Liaoning, China. There are 14 furnaces for smelting magnesia in the plant. The smelting period is from 10:00 pm to 8:00 am every day when the plant can enjoy a lowest electric rate of 0.189 $\mathrm{RMB} / \mathrm{KWh}$. The power demand of one furnace fluctuates between $0.9 \mathrm{MWh}$ and 1.9 MW due to the changing of the operation modes. The CMD of the plant is $22.5 \mathrm{MWh}$ with the average daily energy consumption of $226.8 \mathrm{MWh}$. One furnace has an installed capacity of producing 7 tons of magnesia every day, and the total production output of the plant is required to exceed 80 tons every day. It is also requested that the percentage of magnesia in the final product exceeds $97.7 \%$.

The plant formerly adopted a simple MD control mechanism, which automatically sheds the loads of two randomly selected furnaces when the load surpasses a specified level. The mechanism needs frequently cut off the power supply to furnaces in smelting process, which does harm to the production and facilities. In this paper, we investigate a load scheduling strategy that determines the optimal power consumption by each furnace on each period. The load scheduling results serve as set points to guide the ACM to adjust the actual power consumption by each furnace on each period. An LS-SVM technique is used to estimate the functions of production targets and the modified MOPSO is devised to solve the load scheduling problem. A well-tuned parameters setting for MOPSO is shown in Table I.

\section{TABLE I. THE PARAMETERS SETTING FOR MOPSO}

\begin{tabular}{c|c|c|c|c}
\hline Population & Generations & $\begin{array}{c}\text { Repository } \\
\text { size }\end{array}$ & Division & $\begin{array}{c}\text { Inertia } \\
\text { weight }\end{array}$ \\
\hline 200 & 500 & 200 & 50 & 0.45 \\
\hline
\end{tabular}

\section{B. Results and Analysis}

The LS-SVM based functions of production targets are trained and validated by real data obtained from site measurements. Let's take one function $y_{5 t}=f_{5}\left(p_{5 t}, s_{5 t}\right)$, which represents the estimation of production output of furnace 5 , as an example. The model is trained with 401 groups of data, and validated with 100 groups of data. The model validation results are shown in Fig. 5, which shows that the model precision is satisfactory. The autocorrelation analysis of the model prediction error is conducted and shown in Fig. 6, which further proves that the LS-SVM model is reliable.
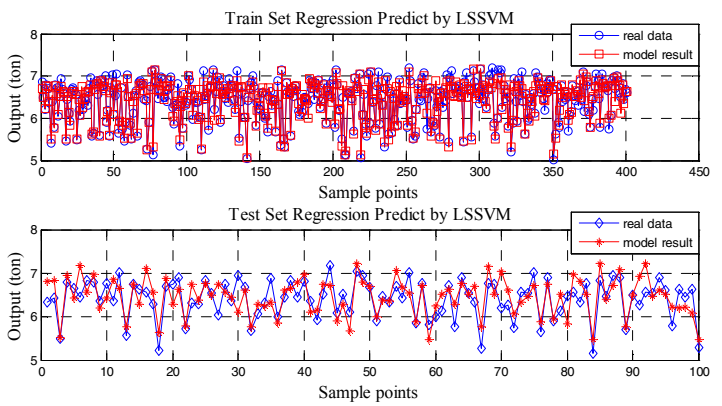

Fig. 5. The comparison of model prediction and real data.

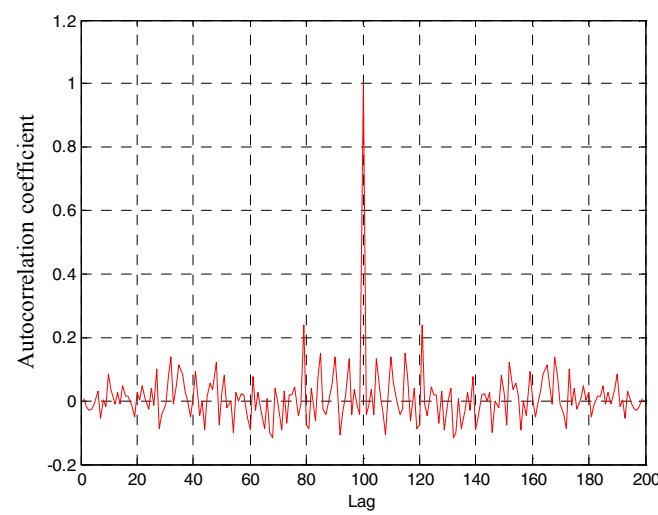

Fig. 6. The comparison of model prediction and real data. 
Solving the load scheduling model by the modified MOPSO, a non-dominated set of solutions is found and the Pareto front of the set of solutions is shown in Fig. 7. From Fig. 7 , it can be seen that some constraint-violated solutions, where their production output is lower than 80 tons, are reserved because they perform excellently on the other production target. These solutions are not only helpful for exploring better solutions but also promising for decision-makers. The constraint-handing strategy proposed here carries out both the identification and conservation of these solutions.

Decision-makers select one solution from the nondominated set according to their preference, and the solution is used for scheduling the load in the smelting process. Unlike the single-objective load scheduling, the load scheduling here achieves the trade-off between the electricity cost and production targets. The comparison is shown in Table II, where the concerned targets are listed in the first column; the performance of simple MD control on these targets is listed in the second column; the performance of single-objective load scheduling for minimizing the electricity cost is listed in the third column; the performance of multiobjective load scheduling is listed in the third column.

From the comparison results, it can be seen that the multiobjective load scheduling approach achieves an increase of about $14.5 \%$ in the production output, an improvement of about $0.46 \%$ in the percentage of magnesia, with a similar electricity cost over the simple MD control approach. This is because the simple MD control only focuses on keeping the MD under the level of CMD without considering its impact on the production targets. Single-objective load scheduling performs the best in terms of saving the electricity cost via optimizing the unique objective, but its performance on production targets just satisfy the constraints. Multiobjective load scheduling aims to achieve the trade-off between the electricity cost and production output. So, it has the best overall performance.

Based on multiobjective load scheduling, the power consumption on each time interval in the plant is shown in Fig. 8 using a bar graph. It can be seen that the resulting MD level is lower than the CMD, so it is possible to save CMD charges by lowering the CMD next month.

To explore a more economical CMD, the load scheduling model with variable CMD is solved by the modified MOPSO. A non-dominated set of solutions is found and the Pareto front of the set is shown in Fig. 9.

One solution is selected from the non-dominated set by the decision-makers, which achieves a saving of about $2.67 \%$ in the electricity cost. According to the solution, the power consump- tion in the plant is shown in Fig. 10. It can be seen that the actual maximum MD level is $20.4 \mathrm{MW}$, a reduction of about $9 \%$ than the current CMD level, so it is promising to save CMD charges by lowering the CMD level next month.

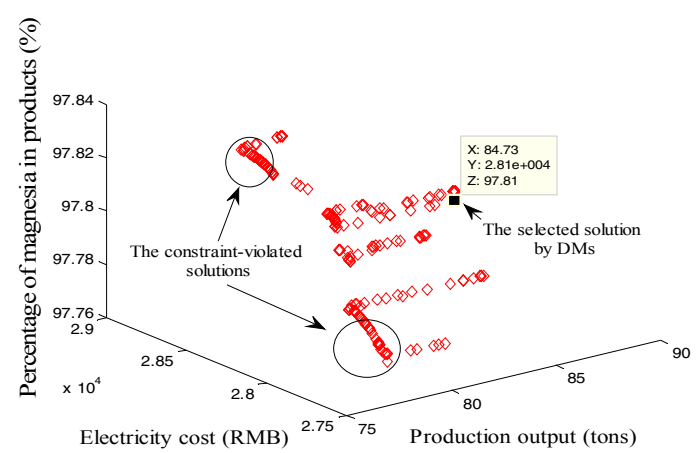

Fig. 7. Pareto front of the non-dominated set found by the modified MOPSO.

TABLE II. THE PERFORMANCE COMPARISON OF THREE APPROACHES

\begin{tabular}{l|c|c|c}
\hline Targets & $\begin{array}{c}\text { Simple MD } \\
\text { control }\end{array}$ & $\begin{array}{c}\text { Single-objective } \\
\text { load scheduling }\end{array}$ & $\begin{array}{c}\text { Multiobjective load } \\
\text { scheduling }\end{array}$ \\
\hline $\begin{array}{l}\text { Production } \\
\text { output }\end{array}$ & 74 & 76 & 84.73 \\
\hline $\begin{array}{l}\text { Percentage of } \\
\text { magnesia }\end{array}$ & $97.36 \%$ & $97.55 \%$ & $97.81 \%$ \\
\hline $\begin{array}{l}\text { Electricity } \\
\text { cost }\end{array}$ & 28217 & 27600 & 28100 \\
\hline
\end{tabular}

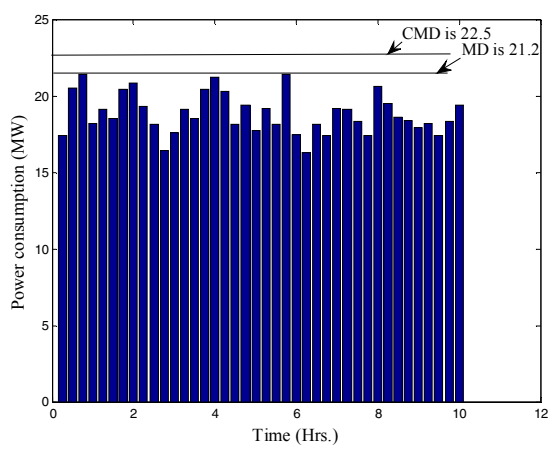

Fig. 8. Power consumption in the plant on each time interval.

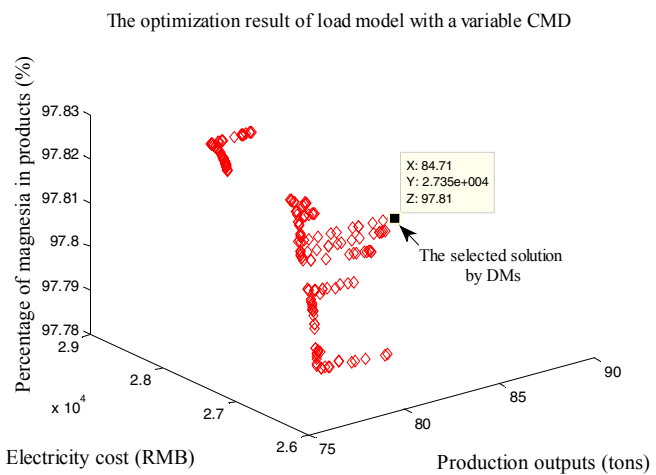

Fig. 9. Pareto front of the non-dominated set found by the modified MOPSO. 


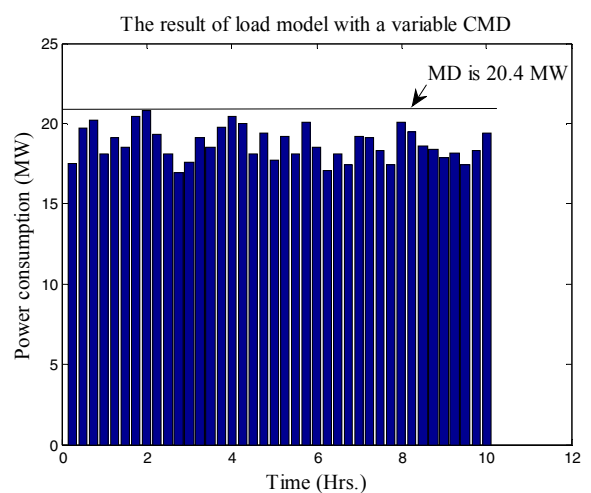

Fig. 10. Power consumption in the plant on each time interval.

\section{CONCLUSION}

A load scheduling model of electric smelting furnaces has been developed based on the LS-SVM technique. By solving this model, both the electricity cost and production targets are taken into account in the design of load scheduling. The multiobjective load scheduling problem is solved by a modified MOPSO algorithm. By means of the algorithm, the past optimization information is inherited to help solve the current optimization problem. A constraint-handing strategy is adopted to efficiently repair the infeasible solutions that violate the MD constraint, and identify and reserve the solutions that violate some constraint of production target but perform excellently on the other production targets. The case study for a typical magnesia-smelting plant shows that multiobjective load scheduling proposed here achieves a trade-off of electricity cost and production targets, so it has a better overall performance than single-objective load scheduling.

\section{REFERENCES}

[1] M. Chaabene, M. B. Ammar and A. Elhajjaji, "Fuzzy approach for optimal energy management of a domestic photovoltaic panel," Applied Energy, vol.84, pp. 992-1001, 2007.

[2] T. Y. Wu, S. S. Shieh, S. S. Jang and C. C .L. Liu, "Optimal energy management integration for a petrochemical plant under considerations of uncertain power supplies," IEEE Trans. Power System, vol. 20, pp. 1431-1439, 2005.

[3] Z. Iou, R. Kumar, J. Sottle, and J. C. Yingling, "An MILP formulation for load side demand control," Electric Machines and Power Systems, vol. 26, pp. 935-949, 1998

[4] S. Ashok and R. Banerjee, "An optimization mode for industrial load management," IEEE Trans. Power System, vol. 16, pp. 879-884, 2001.

[5] S. Ashok, "Peak-load management in steel plants," Applied Energy, vol. 83, pp. 413-424, 2006.

[6] C. A. Babu and S. Ashok, "Peak load management in electrolytic process industries," IEEE Trans. Power System, vol. 23, pp. 399-405, 2008.

[7] S. Ashok and R. Banerjee, "Optimal operation of industrial cogeneration for load management," IEEE Trans. Power System, vol. 18, pp. 931-937, 2003.

[8] A. J. V. Staden, J. F. Zhang and X. H. Xia, "A model predictive control strategy for load shifting in a water pumping scheme with maximum demand charges," Applied Energy, vol. 88, pp. 4785-4794, 2011.
[9] A. Gomes, C. H. Antunes and A. G. Martins, "Design of an adaptive mutation operator in an electrical load management case study," Comp. \& Oper. Res., vol. 35, pp. 2925-2936, 2008.

[10] H. Jorge, C. H. Antunes, and A. G. Martins, "A multiple objective decision support model for the selection of remote load control strategies," IEEE Trans. Power System, vol. 15, pp. 865-872, 2000.

[11] R. Benayoun, I. de Montgolfier, J. Tergny, and O. Laritchev, "Linear programming with multiple objective functions: step method (STEM)," Mathematical Programming, vol. 1, pp. 366-375, 1971.

[12] A. Gomes, C. H. Antunes, A. G. Martins, "A multiple objective evolutionary approach for the design and selection of load control strategies," IEEE Trans. Power System, vol. 19, pp.1173-1180, 2004.

[13] A. Gomes, C. H. Antunes and A. G. Martins, "A Multiple Objective Approach to Direct Load Control Using an Interactive Evolutionary Algorithm," IEEE Trans. Power System, vol. 22, pp. 1004-1011, 2007.

[14] C. Mei, J. Zhou, X. Peng, N. Zhou and P. Zhou, Simulation and Optimization of Furnaces and Kilns for Nonferrous Metallurgical Engineering. New York: Springer, pp. 175-212.

[15] J. A. K. Suykens, J. Vandewalle, "Least squares support vector machine classifiers," Neural Processing Letters, vol. 9, pp. 293-300, 1999.

[16] M. Espinoza, A. K. Johan, Suykens and B. D. Moor, "Fixed-size least squares support vector machines: A large scale application in electrical load forecasting," Computational Management Science, vol. 3, pp. 113129, 2006.

[17] Y. Li, T. Fang and E. Yu, "Short-term electrical load forecasting using least squares support vector machines," Int. Conf. Power Syst. Technol., vol. 1, pp. 230-233, 2002.

[18] W. J. Kong, W. J. Cheng, J. L. Ding and T. Y. Chai, "A reliable and efficient hybrid PSO for parameters optimization of LS-SVM in production rate prediction," in Proc. Int. Symp. on Comput. Intell. and Design, 2010, pp. 140-143.

[19] K. Deb, P. Amrit, A. Sameer and T. Meyrivan, "A fast and elitist multiobjective genetic algorithm: NSGA- II," IEEE Trans. Evol. Comp., vol. 6, pp. 182-197, 2002.

[20] E. Zitzler and L. Thiele, "Multiobjective evolutionary algorithms: A comparative case study and the strength Pareto approach," IEEE Trans. Evol. Comp., vol. 3, pp. 257-271, 1999.

[21] J. D. Knowles and D. W. Corne, "Approximating the nondominated front using the Pareto Archived Evolution Strategy," Evol. Compu., vol. 8, pp. 149-172, 2000

[22] J. Kennedy and R. C. Eberhart, "Particle swarm optimization," in Proc. of IEEE Int. Conf. on Neural Networks, 1995, pp. 1942-1948.

[23] J. Kennedy and R. C. Eberhart, Swarm Intelligence. Morgan Kaufmann Publishers, San Francisco, California, 2001.

[24] J. Moore and R. Chapman, "Application of particle swarm to multiobjective optimization," Department of Computer Science and Software Engineering, Auburn University, 1999.

[25] C. A. C. Coello, D. A. V. Veldhuizen, and G. B. Lamont, Evolutionary Algorithms for Solving Multi-Objective Problems. Kluwer Academic Publishers, New York, May 2002.

[26] C. A. C. Coello, G. T. Pulido, and M. S. Lechuga, "Handling multiple objectives with particle swarm optimization." IEEE Trans. Evol. Comput., vol. 8, pp. 256-279, 2004.

[27] A. Homaifar, S.H.-V. Lai and X. Qi, "Constrained optimization via genetic algorithms," Simulation, vol. 62, pp.242-254, 1994.

[28] J.A. Joines, C.R. Houck, "On the use of nonstationary penalty functions to solve nonlinear constrained optimization problems with GAs," in Proc. of the 1994 Int. Conf. on Evol. Comput.,1994, pp. 579-584

[29] Z. Michalewicz and C. Z. Janikow, "Handling constraints in genetic algorithms," in Proc. of the 4th Int. Conf. on Genetic Algorithms, 1991, pp. $151-157$

[30] Z. Michalewicz and G. Nazhiyath, "Genocop III: A co-evolutionary algorithm for numerical optimization with nonlinear constraints," in Proc. 2nd IEEE Int. Conf. on Evol. Comput., 1995, pp. 647-651. 